\title{
Potential of Alternanthera philoxeroides on Improvement of Anxiety-Like Behavior Induced By Ovariectomized Mice Model
}

\author{
Charinya Khamphukdee ${ }^{1}$, Yaowared Chulikhit ${ }^{2}$, Supawadee Daodee ${ }^{2}$, Orawan Monthakantirat ${ }^{2 *}$ \\ ${ }^{1}$ Graduate School, Faculty of Pharmaceutical Sciences, Khon Kaen University, Khon Kaen, 40002, THAILAND. \\ ${ }^{2}$ Faculty of Pharmaceutical Sciences, Khon Kaen University, Khon Kaen, 40002, THAILAND.
}

\begin{abstract}
Background: Ovariectomy (OVX) causes estrogen deprivation and oxidative stress leads to the hippocampus cell death and affected on mood disorders such as anxiety. Alternanthera philoxeroides was folk used for blood tonic and neuroprotective. Objectives: The action of $A$. philoxeroides extract (AP) on anti-anxiety in OVX mice model was evaluated and chromatographic fingerprint of AP was analyzed. Methods: Mice were ovariectomized to induce estrogen deprivation which involved anxiety. AP (250 and 500 $\mathrm{mg} / \mathrm{kg} / \mathrm{day})$ and $17 \beta$-estradiol $\left(\mathrm{E}_{2}, 1 \mu \mathrm{g} / \mathrm{kg} /\right.$ day $)$ was treated. Six weeks after treatment, anxiety-like behavior was evaluated by the elevated plus maze test (EPM) and light/ dark exploration test (LD). Additionally, fingerprint of AP was characterised by high performance liquid chromatography (HPLC) technique. Results: $E_{2}$ and AP 250 and AP 500 significantly diminished the anxiety behavior. Rutin, quercetin and kaempferol were detected in this plant. Conclusion: AP can manifest anti-anxiety in mice via estrogenic activity and it may action by rutin, quercetin and kaempferol.
\end{abstract}

Keywords: Alternanthera philoxeroides, Ovariectomy, Anxiety, Neuroprotective, Behavior, Flavonoids

\section{INTRODUCTION}

Estrogens are firmly established as regulator of mood such as anxiety and depression in humans and animals. Women suffer from anxiety disorders at twice the rate of men. As life expand of women also has risen so more women will live longer in an $\mathrm{E}_{2}$-deprivation state $^{1-3}$ Estrogen replacement therapy (ERT) in menopausal women is consistently reported to improve mood and learning ${ }^{4}$. Similar effects of $\mathrm{E}_{2}$ on anxiety also have been reported in rodents. OVX indicate behavioral indices of anxiety increase. $\mathrm{E}_{2}$-treated to OVX mice exhibited anxiolytic actions. ${ }^{5}$ For safety concern, ERT come with many undesirable side effects such as thromboembolism. Antianxiety, benzodiazepines, have also been found many side effects such as suicidal ideation. ${ }^{6}$ As a result, there has been increased interest in the use of herbal medicines. We are also interested in herbal plant that has not yet been evaluated scientifically. A. philoxeroides belongs to the family Amaranthaceae. In Ayurvedic and traditional Chinese medicine is used for antiviral, antibacterial in brain, antitumor, antiinflammatory and postnatal complaints such as anxiety. ${ }^{7-10}$ There is no report in antianxiety effect for this potential herb. Our studies aim to evaluate the anxiolytic effect and characterize active compounds from $A$. philoxeroides.

\section{MATERIALS AND METHODS}

Plants materials and preparation of $A$. philoxeroides A. philoxeroides was collected in Khon Kaen province, Thailand. Dry plant was refluxed with ethanol at $50{ }^{\circ} \mathrm{C}$. The combined extract was concentrated by rotary evaporator at $40^{\circ} \mathrm{C}$ and was vaporized by Freeze Dryer.
DOI: 10.5530/ijper.51.3s.73 Correspondence:

Orawan Monthakantirat, Faculty of Pharmaceutical Sciences, Khon Kaen University, Khon Kaen, 40002, THAILAND.

Contact: +66-4320-2305

E-Mail: oramon@kku.ac.th 


\section{Animals}

Female ICR mice (5 weeks old) were obtained from Thai National Laboratory Animal Center. The animals were in a light-controlled room with a $12 \mathrm{~h}$ dark/ light cycle, under controlled temperature $22^{\circ} \mathrm{C} \pm 2{ }^{\circ} \mathrm{C}$, humidity $45 \% \pm 2 \%$. This study was approved by the Animal Ethics Committee of Khon Kaen University. Ovariectomy was performed as previously described ${ }^{11}$. The animals were divided into five groups: (1) sham, (2) OVX, (3) $\mathrm{OVX}+1 \mu \mathrm{g} / \mathrm{kg}$, i.p. $17 \beta$-estradiol, $\left(\mathrm{OVX}+\mathrm{E}_{2}\right)$, (4) $\mathrm{OVX}+250 \mathrm{mg} / \mathrm{kg}$, p.o. of $\mathrm{AP}(\mathrm{OVX}+\mathrm{AP} 250)$, and (5) OVX $+500 \mathrm{mg} / \mathrm{kg}$, p.o. of AP (OVX+AP500). Animals were treated once daily for 6 weeks.

\section{Behavioral analysis}

Elevated Plus Maze: The testing apparatus was consisted of plus-shaped platform elevated $50 \mathrm{~cm}$ from the floor. Two opposite open arms have no wall and two opposite closed arm have $15 \mathrm{~cm}$ tall of wall. The activities of mice were recorded for $5 \mathrm{~min}$. The percentage proportion of entries into open and close arms, and the percentage proportion of time spent in open and close arms were calculated $^{12}$. Light/Dark transition test: The apparatus was partitioned into two chambers, which were bright and darken chamber. The activity of mice were recorded for $5 \mathrm{~min}$. The time spent in dark chamber was calculated as index of anxiety. ${ }^{12}$ Locomotor activity test: Y-maze task was used to determine the locomotor activity. Total arm entries were recorded. ${ }^{12}$

Fingerprint analysis: HPLC separation of the extracts was performed on a Hypersil ODS column and detected by UV at wavelength 254, 275 and $370 \mathrm{~nm}$. Mobile phase composed of $1 \%$ formic acid containing $10 \%$ isopropanol as solvent A and acetonitrile as solvent B. Gradient elution system was operated for the analysis.

\section{Statistical analysis}

Data were expressed as the mean \pm S.E.M. and were examined one-way ANOVA followed by the Tukey test for multiple comparisons among different groups. Differences of $\mathrm{p} \leq 0.05$ were considered to be statistically significant. SigmaStat ${ }^{\circledR}$ ver. 3.5 (SYSTAT Software Inc., Richmond, CA, USA) was used.

\section{RESULTS AND DISCUSSION}

Elevated plus maze: Spontaneously rodents avoid the open, bright and elevated arms of the maze and prefer to stay in the more dark or closed arms. A reduction in anxiety is indicated by an animal's tendency to spend more time interacting with their environment through exploration of the maze that leads to their entering and spending more time in the open arms of the maze. In the elevated plus maze, OVX mice treated with $\mathrm{E}_{2}$, AP250 and AP500 displayed significantly fewer anxiety related behaviors by increasing numbers of entries onto the open arms and reducing numbers of entries into close arms $(P<0.01)$ (Figure $1 \mathrm{~A})$. All treatments increased time spent on the open arms $(P<0.01)$ (Figure 1B) compared with OVX groups. Light/Dark transition test: Consistent with our results in EPM, OVX mice treated with $\mathrm{E}_{2}, \mathrm{AP} 250$ and AP500 showed significantly less anxious behavior in the light/dark apparatus. AP250 and AP500 spent more time in light compartment and fewer spent time in dark chamber $(P<0.01)$ in dose dependent manner (Figure 2). In order to exclude the false positive results from hopeless behavioral tests, locomotor activity test was conducted. It was exhibited that no effect of locomotor activity induced by AP and $\mathrm{E}_{2}$ (Figure 3). Moreover, result from HPLC revealed that some flavonoids such as rutin, quercetin and kaemp-

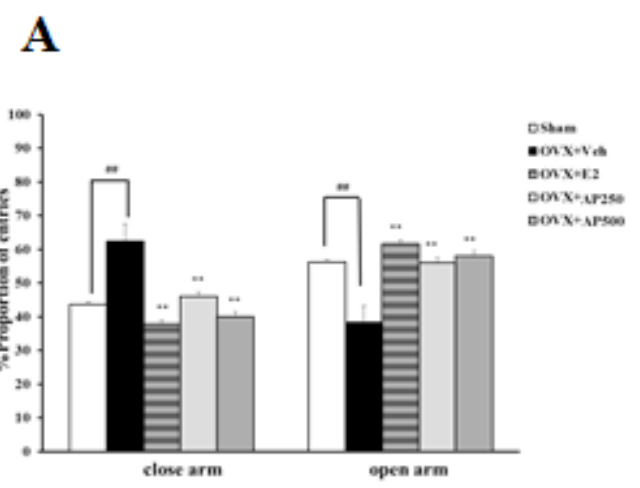

\section{B}

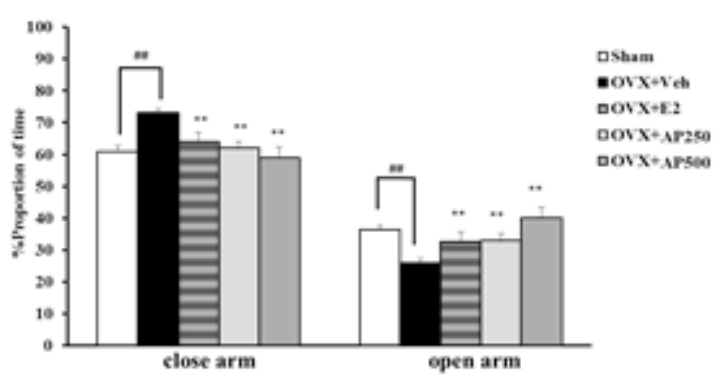

Figure 1: The effect of $\mathrm{E}_{2}$ and $A$. philoxeroides on anxiety in sham and ovariectomized mice using elevated plus maze test. A) The percentage proportion of entries into close and open arms were displayed. B) The percentage proportion of time spent in close and open arms. Values given were the mean \pm S.E.M $(n=8-10)$. Significant ANOVA effect were represented by \#\#p<0.001 vs. sham, ${ }^{* *} p<0.001$ vs. ovariectomized mice. 


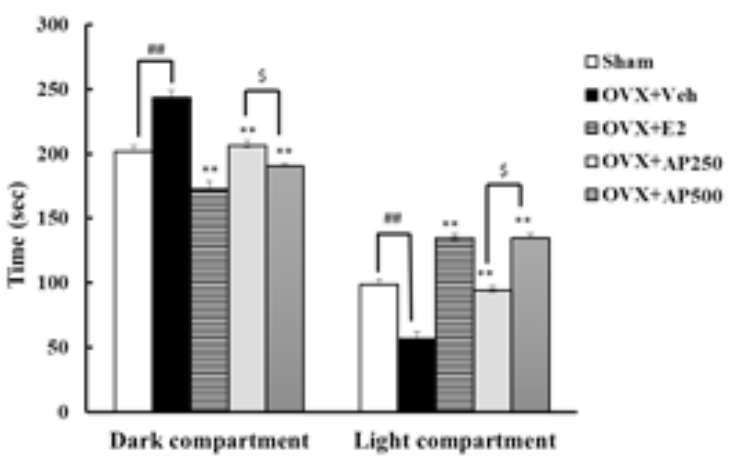

Figure 2: The effect of $\mathrm{E}_{2}$ and $A$. philoxeroides on anxiety in sham and ovariectomized mice using light/dark transition test. The time spent in dark chamber was evaluated. Values given were the mean \pm S.E.M $(n=8)$. Significant ANOVA effect were represented by \#\#p<0.001 vs. sham, ${ }^{*} p<0.001$ vs. ovariectomized mice and $\$ p<0.05$ vs. A. philoxeroides.

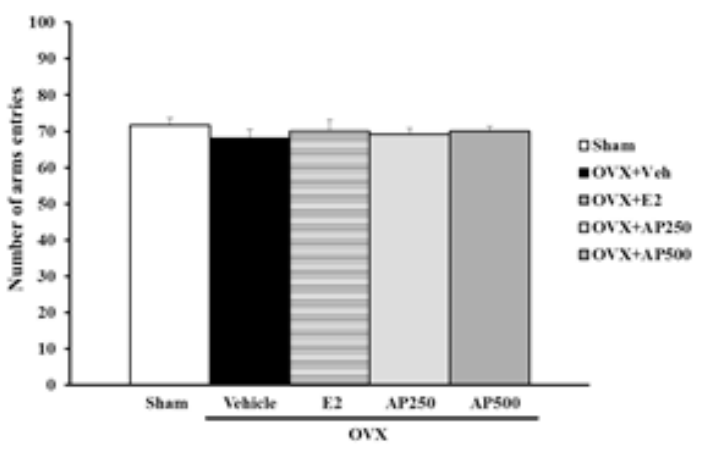

Figure 3: The effect of daily administration of $\mathrm{E}_{2}$ and $A$. philoxeroides extract on locomotor activity in Y-maze test. Number of arms entries of each animal group was determined. Each data column represents the mean \pm S.E.M $(n=$ 8-10 in each animal group).

ferol were detected and the content was approximately $0.4 \mathrm{mg} / \mathrm{g}$ extract per each. These fingerprint chromatograms showed the specific characteristic of AP which could be used for controlling the quality of this plant. Some literatures reported the neuroprotective also antianxiety action of these found flavonoids. ${ }^{13-14}$ However, it will be necessary in subsequent studies the relevant mechanism such as estrogen receptor, $\gamma$-aminobutyric acid A receptor, serotonin receptor and hypothalamicpituitary-adrenal.

\section{CONCLUSION}

These finding suggested that $A$. philoxeroides significantly reduced anxiety behavior investigated by EPM test and LD transition test in OVX mice model. The action may affect from flavonoids such as rutin, quercetin and kaempferol that found in this plant. Thus A. philoxeroides has potential to develop for antianxiety especially menopausal women.

\section{ACKNOWLEDGEMENT}

The authors are grateful for Thailand Research Fund for the Research Funding (RDG5520030).

\section{CONFLICT OF INTEREST}

The authors declare that they have no conflict of interests.

\section{ABBREVIATION USED}

AP: Alternanthera philoxeroides; $\mathrm{E}_{2}$ : 17ß-Estradiol; EPM: Elevated plus maze; ERT: Estrogen replacement therapy; LD: Light/Dark transition test; OVX: Ovariectomy.

\section{REFERENCES}

1. Palanza P. Animal models of anxiety and depression: how are females different? Neurosci Biobehav Rev. 2001;25(3):219-233.

2. Pigott TA. Anxiety disorders in women. Psychiatr Clin North Am. 2003;26:621-72.

3. Steiner M, Dunn E, Born L. Hormones and mood: from menarche to menopause and beyond. J Affect Disord. 2003;74(1):67-83.

4. Leret ML, Molina-Holgado F, Gonzalez MI. The effect of perinatal exposure to estrogens on the sexually dimorphic response to novelty. Physiol Behav. 1994;55(2):371-3.

5. Mora S, Dussaubat N, Diaz-Veliz G. Effects of the estrous cycle and ovarian hormones on behavioral indices of anxiety in female rats. Psychoneuro endocrinology. 1996;21(7):609-20.

6. Lakhan SE, Vieira KF. Nutritional and herbal supplements for anxiety and anxiety-related disorders: systematic review. Nutrition Journal. 2010;9(1):42-56.

7. Li B, Guo Q, Tian Y, Liu S, Wang Q, Chen L, et al. New Anti-HBV C Boivinopyranosyl Flavones from Alternanthera philoxeroides. Molecules 2016;21;33-40.

8. Jogendra HC, Avinash PV, Mohan KV, Patil DA and Ravindra MG. A current update on phytopharmacology of the genus Alternanthera. Journal of Pharmacy Research 2012;5(4);1924-9.

9. Fang J, Jia W, Gao W, Yao Z, Teng J, Zhao A, Duan H. Antitumor constituents from Alternanthera philoxeroides. Journal of Asian Natural Products Research. 2007;9(6):511-5.

10. Rahman AHMM, and Gulshana MIA. Taxonomy and Medicinal Uses on Amaranthaceae Family of Rajshahi, Bangladesh. Applied Ecology and Environmental Sciences. 2014;2(2):54-59.

11. Monthakantirat $\mathrm{O}$, Sukano W, Umehara K, Noguchi H, Chulikhit $\mathrm{Y}$, Matsumoto $\mathrm{K}$. Effect of miroestrol on ovariectomy-induced cognitive impairment and lipid peroxidation in mouse brain. Phytomedicine. 2014;21(11):1249-55.

12. Vogel HG. (Ed). (2008). Drug Discovery and Evaluation: Pharmacological APsays. $3^{\text {rd }}$. New York, United State: Springer-Verlag Berlin Heidelberg.

13. Hernandez-Leon A, González-Trujano ME, Fernández-GuAPti A. The anxiolytic-like effect of rutin in rats involves GABAA receptors in the bAPolateral amygdala. Behav Pharmacol. 2017;28(4):303-12.

14. Mohammad HF, Roodabeh B, Roja R, Faezeh A, Mohammad A. A Systematic Review of Plant-Derived Natural Compounds for Anxiety Disorders. Current Topics in Medicinal Chemistry. 2016;16(17):1924-42. 


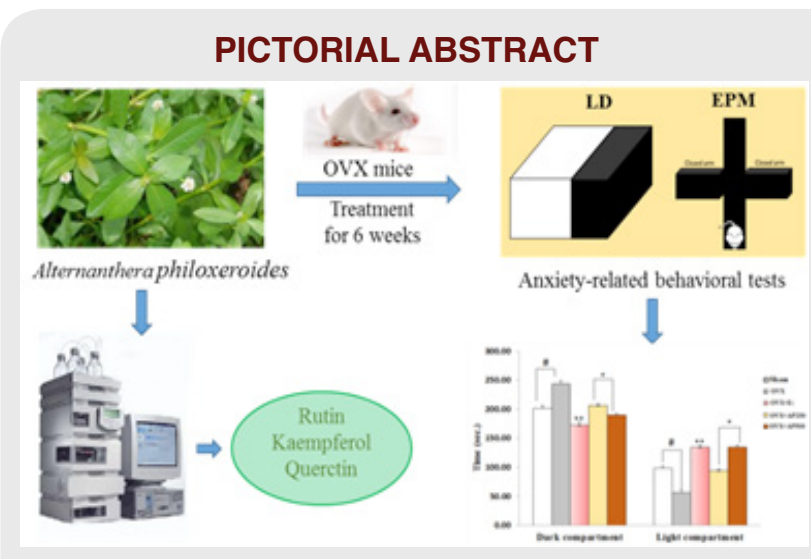

\section{ABOUT AUTHORS}

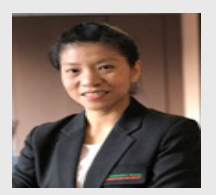

Dr. Orawan Monthakantirat: She is working in Division of Pharmaceutical Chemistry, Faculty of Pharmaceutical Sciences, Khon Kaen University. She has experience in the area of pharmaceutical chemistry, the medicinal plants, and phytochemistry. She has published a number of papers in her expertise area in various journals.

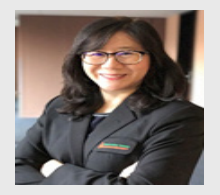

Dr. Yaowared Chulikhit: She is working as Assistant Professor in Division of Pharmaceutical Chemistry, Faculty of Pharmaceutical Sciences, Khon Kaen University. She has experience in the area of pharmaceutical chemistry, neuropharmacology, and molecular biotechnology. She has published a number of papers in her expertise area in various journals.

Cite this article: Khamphukdee C, Chulikhit Y, Daodee S, Monthakantirat O. Potential of Alternanthera philoxeroides on Improvement of Anxiety-Like Behavior Induced By Ovariectomized Mice Model. Indian J of Pharmaceutical Education and Research. 2017;51(3)Suppl:S494-97. 